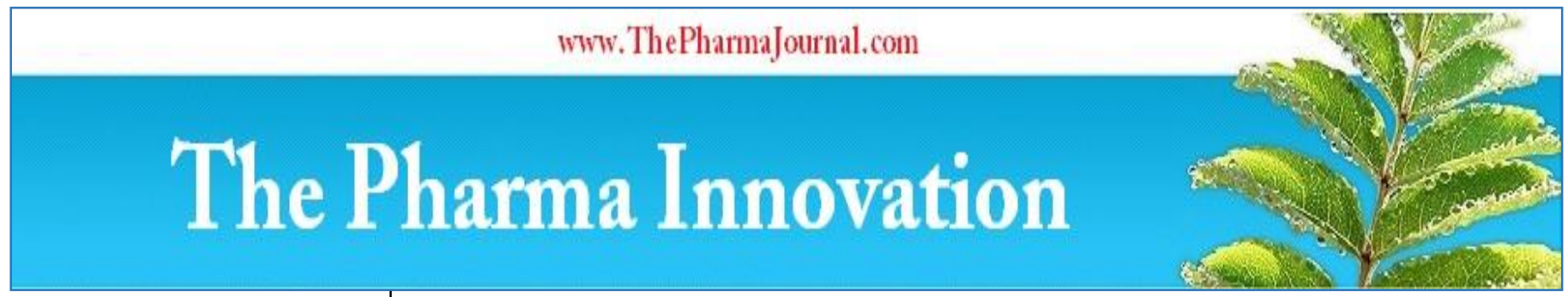

ISSN (E): 2277 - 7695

ISSN (P): 2349-8242

NAAS Rating: 5.23

TPI 2021; 10(3): 35-44

(C) 2021 TPI

www.thepharmajournal.com

Received: 15-01-2021

Accepted: 19-02-2021

Payal Tripathi

Department of Forensic Science, Institute of Science, Banaras

Hindu University, Varanasi,

Uttar Pradesh, India

\section{The adverse effect of toxic plant constituent found in India: Forensic approach}

\section{Payal Tripathi}

\author{
DOI: $\underline{\text { https://doi.org/10.22271/tpi.2021.v10.i3a.6072 }}$
}

\begin{abstract}
There are various plant originated active chemical constituents which are toxicologically significant includes proteins, phenolic compounds, alkaloids, glycosides, and resins, etc. Out of these huge numbers of plants in the environment, few cause acute toxicity, severe illness if it is consumed. The diversity of active chemical constituent in plants is quite amazing. Natural poisons are those chemicals that kill without violence, mysteriously, secretly destroy life. Some of the common plant families and its toxic constituent are easily available like Euphorbiaceae (cleistanthin, toxalbumin, curcin), Solanaceae (capsicin, atropine, dutarin), Apocyanacae (uscharin, odolotoxin, neriodorin), Leguminosae (cytisine sparteine), Fabaceae (abrasine, diaminopropionic acid), Papaveraceae (narcotine, dihydrosangunarine). The natural poisons are also used by criminals for stupefying people that facilitate robbery, murder and other cases. These natural poisons are readily accessible and very cheap, so skilful poisoners prefer this toxic plant for a crime. In this work author revised literature related to the classification of plant's chemical constituents, its lethal dose and metabolic effects on the body. It has been thoroughly received and collected from journals and textbooks to make this review useful to all specialists of different discipline and it also has significant forensic importance.
\end{abstract}

Keywords: Natural poison, bioterrorism, alkaloids, toxic resin, biological significance

\section{Introduction}

A natural poison is the substances, which has the ability to produce adverse effect or death when it is absorbed by the body. Appropriate dosages can differentiate poison and also the remedial measures. All chemicals can produce injury, adverse effect or death under certain physiological conditions. Moreover, they are able to produce detrimental effects on a living organism. As a result, there may be a change in the structure of the substance or functional processes, which may produce injury or even death ${ }^{[1,2]}$. In the court of law, the major difference between medicine and poison is the intention with which it is given. If any active chemical constituent has given for purpose to save a life, it is medicine but if it is given with the purpose to cause damage to health, it is a poison ${ }^{[3]}$. The father of toxicology Methieu Orfila (1787-1853) said in the sixteenth century, "Everything is poison, there is poison in everything, only the dose makes a thing not a poison ${ }^{[4]}$. An ideal poison should be cheap, easily available, colorless, odorless, tasteless, highly toxic, capable of painless death, signs and symptoms should resemble a natural death, capable of being administered easily either in food/ drink/ medicine and must be rapidly cleared from the victim's body that it could be made undetectable ${ }^{[5]}$. The toxic plants could be further classified by their origin (a) species of plants, bacteria, fungi, protists, and animals (natural poisons), (b) sedative drugs, chemicals, pesticides, alcohols and household poisons (synthetic chemicals) ${ }^{[6]}$. The prevalence of criminal poisoning in India among the topmost in the world and every year upwards 50000 people pass away because of toxic exposure ${ }^{[7]}$. The major causes of poisoning are many civilian, industrial, accidental and deliberate ${ }^{[8]}$. The toxic chemical compounds may be a single compound or a number of compounds in the same plants. The wide range of active chemical constituents of plant kingdom can be classified as primary metabolites and secondary metabolites. Toxic constituents in plants are secondary metabolites. Above 5000 species of toxic plants growing as herbs, shrubs and trees in the world. The plant product, proteins, enzymes, active chemical constituents such as alkaloids, glycosides, saponins, resinoids, oxalates photosensitizing compounds and mineral compounds are accumulated from the soil. Moreover, the overdose of plant extracts has adverse effect or acts as poisons. Plants containing alkaloids often produce unpleasant or dangerous reactions in the nervous system. Examples are paralysis (Conium maculatum), hallucinations (Datura stramonium) or cardiac

\footnotetext{
Corresponding Author: Payal Tripathi

Department of Forensic Science,

Institute of Science, Banaras

Hindu University, Varanasi,

Uttar Pradesh, India
}

$$
\sim 35 \sim
$$


arrest (Taxus baccata). Generally, use of plant poisons in suicide, homicide, robbery and other crimes are common in the world as many poisonous plants grow in wild, e.g. Ricinus, Jatropa, Datura, Oleanders, and Nux vomica etc. Many articles have been reported on the principle of plant toxicology, but no work has been done especially on the study of active chemical constituents of plants in terms of the forensic context.

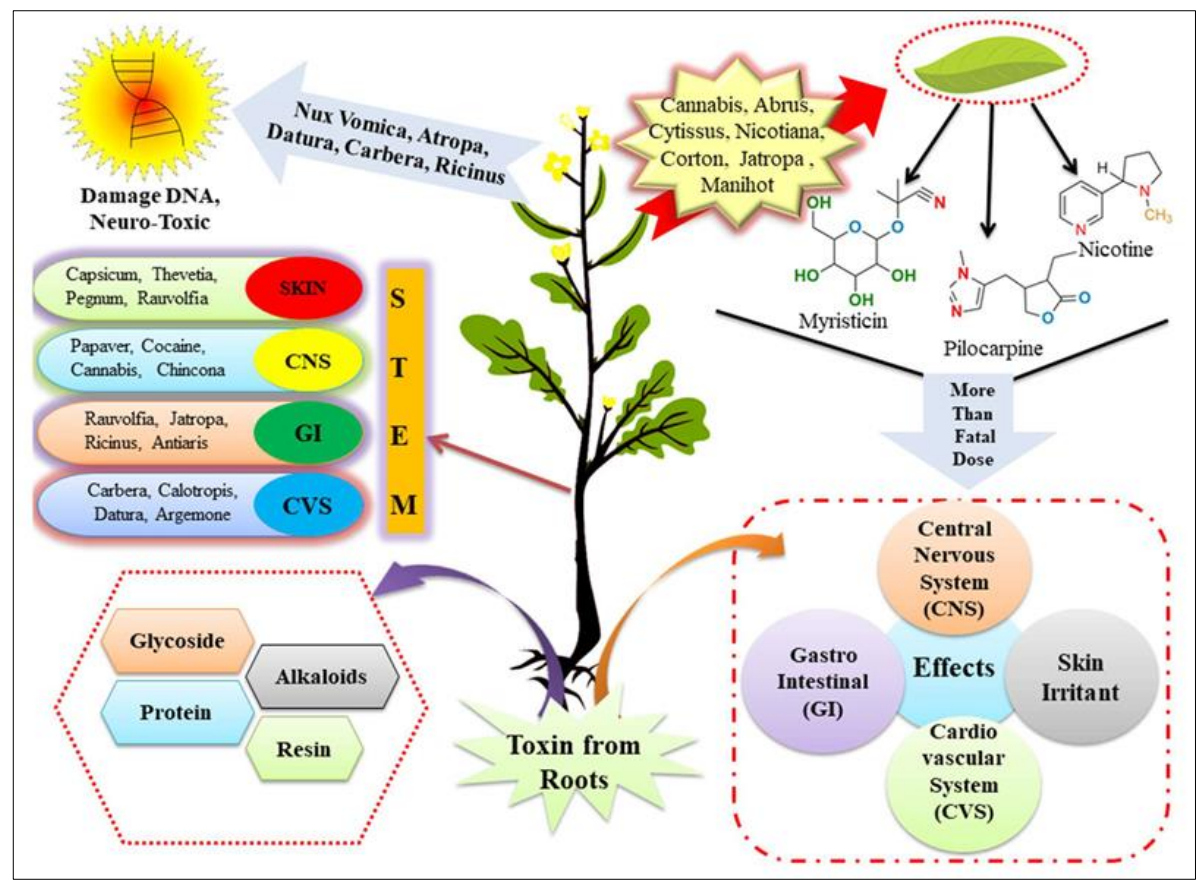

Graphical Abstract

\subsection{Criminal Offences in Indian Panel Code (IPC)}

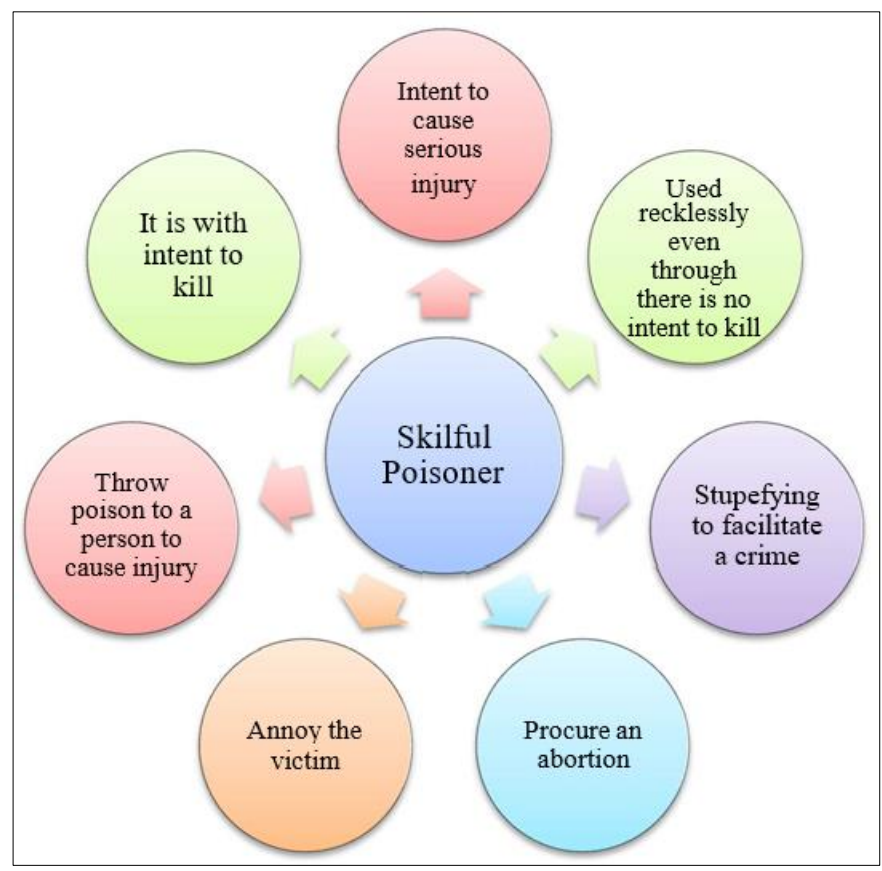

Fig 1: The strategy of poison in criminal cases by a skilful poisoner

The major section of Indian penal code (1860) comes under adulteration of food and use of toxic chemical are IPC section $272,273,274,276,277,278,328$. At present day's toxic plants such as Datura, Nerium, Atropa, and Corton are frequently used for robbery, murder and suicidal purposes. Cases of robbery (in bus, trains), using stupefying poisons such as Datura, Jatropha, Cannabis is very common in cities. Such chemical constituent has been frequently detected in the vomit of the victims. Capsicum annum (chili) powder is commonly used in the robbery or a confession of some guilt by introducing it into the nostrils, eyes, urethra, vagina, or rectum. Conium maculatum (Hemlock) is an Athenian state of poison used for the death of Socrates. Hyoscyamus niger is used in war to control shell-shock. In Crippen cases, the overdose of plants active constituent act as poisons. In the field of forensic science, the active constituent of toxic plants acts as a truth serum. 


\subsection{Distribution of Toxic Plants in India}

India has a vast occurrence of forests like tropical forest, temperate forest, evergreen forest, Western Ghats from where the toxic plants belong. Generally, the toxic plants belong to the families of Euphorbiaceae, Solanaceae, Fabaceae, Apocyanacae and a host of other toxic families. The active constituents of plants contain Crotonic acid, Tiglinic acids, Cleistanthin, Curcin, Cyanogenic Glycoside, Atropine, Scopolamine, Hyoscyamine, Belladonnine, Solanine Steroidal Glycoside, Cerberin, Cerberoside, Odollin, Odolotoxin, Thevetin and Cerapain types of chemical. These plants mainly cover the northeastern part of Uttar Pradesh, Madhya Pradesh and coastal part of western India. Lantana camara, Mucuna pruriens, Solanum virginianum, Erythrina stricta, Crotalaria pallida, Cassia alata, Arisaema tortuosum, Datura innoxia, Cestrum nocturnum, Amorphophallus paeoniifolius are found in Western Himalaya ascending to 1350-4350 meter altitude.
The Himalayan belt includes Chlorophytum breviscapum, Polygonatum brevistylum, Smilacina oleracea and Tupistra nutans to altitudes between ranges of 350-4350 meter. From Kashmir to Sikkim at an altitude of 4000-5550 meter Lantana camara, Ipomea batatas, Nerium olender are usually found. Alangiaceae, Balanitaceae, Cuscutaceae, Dioscoreaceae, Liliaceae, Ulmaceae, Meliaceae occurs in the Deccan Peninsula and Central India. Papilionaceae, Cannabaceae, Apocynaceae, Cucurbitaceae, Euphorbiaceae, Dioscoreaceae are distributed in the South of Deccan in Nilgiri at 550-900 meter altitudes. Ricinus communis, Croton tiglium, Atropa belladonna, Capsicum annum, Datura fastuosa, Calotropis gigentea, Abrus precatorius, Argemone mexicana are distributed throughout India. The review of all the toxic plants summarised in table 1 according to their toxic constituent, toxic parts, that provide a fundamental database for the forensic community.

Table 1: List of toxic plants with their families, toxic parts, the active constituent, fatal dose and fatal period

\begin{tabular}{|c|c|c|c|c|c|c|c|}
\hline Name of Plant & Family* & Toxic Parts & Toxic Constituents & Fatal Dose & $\begin{array}{l}\text { Fatal } \\
\text { period }\end{array}$ & Chemical constituents & Ref \\
\hline Cleistanthus collinus & Eup & Leafs, Bark & $\begin{array}{c}\text { phyllamyricin, justicidin } \\
\text { B, Diphyllin }\end{array}$ & $0.5 \mathrm{mg} / \mathrm{kg}$ (animals) & 3-7 days & Cleistanthins & {$[9,10]$} \\
\hline $\begin{array}{c}\text { Croton tiglium } \\
\text { (Jamal-gota) }\end{array}$ & Eup & Seed, oil & $\begin{array}{c}\text { Tiglinic acids, Crotonic } \\
\text { acid }\end{array}$ & $\begin{array}{c}4-6 \text { seeds, } \\
1-2 \text { mL of oil }\end{array}$ & $\begin{array}{c}6 \mathrm{~h} \text { to } 3 \\
\text { days }\end{array}$ & Toxalbumines & [11] \\
\hline $\begin{array}{l}\text { Euphorbia } \\
\text { helioscopia } \\
\text { (Sun spurge) }\end{array}$ & Eup & Milky latex & $\begin{array}{c}\text { Non-Haemolytic Saponin } \\
\text { and } \\
\text { Phasin }\end{array}$ & $2 \mathrm{~g} / \mathrm{kg}$ & $\begin{array}{c}2-3 \\
\text { months }\end{array}$ & Diterpenoid & {$[12,13]$} \\
\hline $\begin{array}{l}\text { Jatropha } \\
\text { multifida } \\
\text { (Bherenda) }\end{array}$ & Eup & Foliage, fruits & Curcin & $>30 \mathrm{mg} / \mathrm{kg}$ & $\begin{array}{c}15-20 \\
\text { days }\end{array}$ & Diterpenoid & [14] \\
\hline $\begin{array}{l}\text { Manihot esculenta } \\
\text { (Sakarkanda) }\end{array}$ & Eup & Tubers & Cyanogenetic glycosides & $1 \mathrm{mg} / \mathrm{kg}$ & 2-4 hours & Cyanogenic Glycoside & [15] \\
\hline $\begin{array}{l}\text { Manihot utilissima } \\
\text { (Cassava, tapioca) }\end{array}$ & Eup & Root, leafs & $\begin{array}{l}\text { Linamarin a Cyanogenic } \\
\text { Glycoside }\end{array}$ & $\begin{array}{l}300 \mathrm{~g} \text { (adult) } \\
125 \mathrm{~g} \text { (child) }\end{array}$ & 4-6 hours & $\begin{array}{c}\text { Cyanophoric, } \\
\text { Cyanogenetic glycosides }\end{array}$ & [16] \\
\hline $\begin{array}{l}\text { Ricinus communis } \\
\text { (Erandi) }\end{array}$ & Eup & Entire plant & Ricin and Ricinine & $\begin{array}{l}6 \mathrm{mg} \text { of ricin } \\
(10 \text { seeds })\end{array}$ & $\begin{array}{l}2 \text { to } \\
\text { several } \\
\text { Days }\end{array}$ & $\begin{array}{l}\text { Pyridine-piperidine } \\
\text { alkaloid }\end{array}$ & {$[17,18]$} \\
\hline $\begin{array}{l}\text { Atropa belladonna } \\
\text { (Deadly nightshade) }\end{array}$ & Sola & All parts & $\begin{array}{c}\text { Atropine, Scopolamine, } \\
\text { Hyoscyamine, } \\
\text { Belladonnine }\end{array}$ & $\begin{array}{l}120 \mathrm{mg} \text { (atropine) } \\
30 \mathrm{mg} \text { (hyoscine) }\end{array}$ & $24 \mathrm{~h}$ & Tropane alkaloid & {$[19,20]$} \\
\hline $\begin{array}{c}\text { Capsicum annum } \\
\text { (Chillies and Mirch) }\end{array}$ & Sola & Fruit & Capsaicin, Capsicin & $110-150 \mathrm{mg} / \mathrm{kg}$ (oral) & 6-7 days & Alkaloids & [21] \\
\hline $\begin{array}{c}\text { Datura fastuosa } \\
\text { (Thorn apple and } \\
\text { datura) }\end{array}$ & Sola & $\begin{array}{c}\text { All parts } \\
\text { especially } \\
\text { seeds and fruit } \\
\end{array}$ & $\begin{array}{c}\text { Atropine, Hyoscyamine, } \\
\text { Hyoscine } \\
\text { and Dutarin } \\
\end{array}$ & $0.6-1$ gram & $24 \mathrm{~h}$ & Tropane alkaloid & [22] \\
\hline $\begin{array}{c}\text { Hyoscyamus niger } \\
\text { (Henbane) }\end{array}$ & Sola & All parts & $\begin{array}{l}\text { Atropine, Hyoscine and } \\
\text { Hyoscyamine }\end{array}$ & $125 \mathrm{mg}$ (hyoscyamine) & $24 \mathrm{~h}$ & $\begin{array}{c}\text { Alkaloid } \\
\text { Hyoscyamine }\end{array}$ & [23] \\
\hline $\begin{array}{l}\text { Nicotiana tabacum } \\
\text { (Tobacco and } \\
\text { tambaku) }\end{array}$ & Sola & $\begin{array}{l}\text { All parts } \\
\text { except ripe } \\
\text { seeds }\end{array}$ & Nicotine & $60-100 \mathrm{mg}$ & $5-15 \min$ & $\begin{array}{l}\text { Senecio alkaloids } \\
\text { (Pyrrolidine group) }\end{array}$ & {$[24,25]$} \\
\hline $\begin{array}{l}\text { Solanum nigrum } \\
\text { (Black nightshade) }\end{array}$ & Sola & $\begin{array}{l}\text { Immature } \\
\text { berries }\end{array}$ & Solanine and Steroids & $3-6 \mathrm{mg} / \mathrm{kg}$ & $8-12 \mathrm{~h}$ & $\begin{array}{l}\text { Pyridine-piperidine } \\
\text { alkaloids }\end{array}$ & [26] \\
\hline $\begin{array}{c}\text { Calotropis gigantea } \\
\text { (Madar, akdo) }\end{array}$ & Apocy & Juice, roots & $\begin{array}{c}\text { Uscharin, Calotropin, } \\
\text { Calactin }\end{array}$ & $0.12 \mathrm{mg} / \mathrm{kg}$ calotropin & 12 to $24 \mathrm{~h}$ & $\begin{array}{l}\text { Steroidal glycosides } \\
\text { Cardiac glycosides }\end{array}$ & [27] \\
\hline $\begin{array}{l}\text { Cerbera odollam } \\
\text { (Dabur) }\end{array}$ & Apocy & Fruit, seed & $\begin{array}{c}\text { Cerberin, Cerberoside, } \\
\text { Odolin, } \\
\text { Thevetin }\end{array}$ & Kernel of one fruit & $\begin{array}{c}1 \text { - } 2 \text { days } \\
\text { or } \\
\text { more }\end{array}$ & $\begin{array}{l}\text { Steroidal glycosides } \\
\text { Cardiac glycosides }\end{array}$ & [28] \\
\hline $\begin{array}{c}\text { Cerbera thevetia } \\
\text { ( Pila } \\
\text { kaner) }\end{array}$ & Apocy & $\begin{array}{c}\text { All parts } \\
\text { especially } \\
\text { leaves \& fruits }\end{array}$ & $\begin{array}{l}\text { Thevetin, Thevetoxin, } \\
\text { Nerifolin, Peruvoside }\end{array}$ & $\begin{array}{l}8-10 \text { seeds, } 15-20 \mathrm{~g} \text { of } \\
\text { the root, } 5 \text { to } 10 \text { leaves }\end{array}$ & $\begin{array}{l}\text { Depend } \\
\text { upon } \\
\text { Quantity }\end{array}$ & $\begin{array}{l}\text { Steroidal glycosides } \\
\text { Cardiac glycosides }\end{array}$ & [29] \\
\hline $\begin{array}{l}\text { Nerium odorum } \\
\text { (Kaner) }\end{array}$ & Apocy & All parts & $\begin{array}{l}\text { Neriodorin, Neriodorein, } \\
\text { Oleandrin }\end{array}$ & $15-20 \mathrm{~g}$ root & $\begin{array}{l}24-36 \\
\text { hours }\end{array}$ & $\begin{array}{l}\text { Steroidal glycosides } \\
\text { Cardiac glycosides }\end{array}$ & [30] \\
\hline Rauwolfia serpentina & Apocy & Roots & Reserpine, Serpentine & $200-300 \mathrm{mg} /$ day & 2-3 weak & $\begin{array}{l}\text { Steroidal glycosides } \\
\text { Cardiac glycosides }\end{array}$ & [31] \\
\hline
\end{tabular}




\begin{tabular}{|c|c|c|c|c|c|c|c|}
\hline $\begin{array}{c}\text { Thevetia peruviana } \\
\text { (Suicide tree Pila } \\
\text { kaner) }\end{array}$ & Apocy & $\begin{array}{l}\text { Seeds and } \\
\text { milky } \\
\text { juice }\end{array}$ & $\begin{array}{l}\text { Thevetin A and Thevetin } \\
\text { B }\end{array}$ & $\begin{array}{c}\text { Kernel of } 1 \text { - fruit, } \\
7-8 \text { leaf }\end{array}$ & $24-36 \mathrm{~h}$ & $\begin{array}{l}\text { Steroidal glycosides } \\
\text { Cardiac glycosides }\end{array}$ & [32] \\
\hline $\begin{array}{c}\text { Crotalaria } \\
\text { spectabilis } \\
\text { (Jhunjhunia) }\end{array}$ & Legu & seeds & Mono-crotaline & 65 mg/kg (chicken) & $24 \mathrm{~h}$ & Pyrrolizidine alkaloids & [33] \\
\hline $\begin{array}{l}\text { Cytisus scoparius } \\
\text { (Yellow broom) }\end{array}$ & Legu & $\begin{array}{l}\text { Seed, leaves } \\
\text { and } \\
\text { twigs }\end{array}$ & Cytisine and Sparteine & & & $\begin{array}{c}\text { Pyridine-piperidine } \\
\text { alkaloids }\end{array}$ & [34] \\
\hline $\begin{array}{l}\text { Mucuna prurita } \\
\text { (Velvet bean) }\end{array}$ & Legu & Seeds & $\begin{array}{l}\text { Mucunain, } \\
\text { Serotonin }\end{array}$ & $2 \mathrm{~g} / \mathrm{kg}$ & $\begin{array}{c}10-14 \\
\text { days }\end{array}$ & $\begin{array}{l}\text { Imidazole } \\
\text { Alkaloids }\end{array}$ & [35] \\
\hline $\begin{array}{l}\text { Abrus precatorius } \\
\text { (Crab's eyes, Gunji) }\end{array}$ & Legu & $\begin{array}{c}\text { Roots, seeds } \\
\text { and } \\
\text { leaves } \\
\end{array}$ & Abrin, Abrine & $\begin{array}{c}1 \text { - } 2 \text { seed, } \\
90-120 \mathrm{mg} / \mathrm{kg} \text { (Abrin) }\end{array}$ & $3-5$ days & Toxalbumines & [36] \\
\hline $\begin{array}{c}\text { Lathyrus sativus } \\
\text { (Gross pea, Khesari) }\end{array}$ & Faba & Seeds & $\begin{array}{c}\beta \text {-Cyanoalanine, } 2,4- \\
\text { diaminobutyric acid, } \\
\text { Selenium }\end{array}$ & $1-10 \mathrm{mg} / \mathrm{kg}$ & $3-5$ days & Cyanophoric glycosides & [37] \\
\hline $\begin{array}{l}\text { Argemone Mexicana } \\
\text { (Sial-kanta) }\end{array}$ & Papa & $\begin{array}{l}\text { All parts } \\
\text { especially } \\
\text { seeds }\end{array}$ & Dihydro-Sanguinarine & $10 \mathrm{mg} / \mathrm{kg}$ & & Isoquinoline alkaloids & $\begin{array}{l}{[38-} \\
40]\end{array}$ \\
\hline $\begin{array}{l}\text { Papaver somniferum } \\
\text { (Opium poppy) }\end{array}$ & Papa & \begin{tabular}{|} 
Ripe and dried \\
capsules, \\
petals \\
and seeds \\
\end{tabular} & $\begin{array}{c}\text { Papaverine, narcotine, } \\
\text { narceine }\end{array}$ & $\begin{array}{c}2 \text { gm (opium) } \\
0.2 \mathrm{gm} \text { (mor-phine) }, 0.5 \\
\text { gm (codeine) }\end{array}$ & 2 to $6 \mathrm{~h}$ & Steroidal alkaloids & [41] \\
\hline $\begin{array}{c}\text { Lobelia } \\
\text { nicotianifolia } \\
\text { (Indian lobelia and } \\
\text { dhawal) }\end{array}$ & Cam & All parts & Lobeline & $\begin{array}{c}10 \mathrm{mg} \text { (Lobeline), } \\
3.75 \mathrm{~g} \text { (leafs) }\end{array}$ & $\begin{array}{l}30 \text { min to } \\
\text { a day }\end{array}$ & Alkaloids & [42] \\
\hline $\begin{array}{c}\text { Plumbago zeylanica } \\
\text { (Ceylon leadwort } \\
\text { and chitrak) }\end{array}$ & $\mathrm{Plu}$ & Root & Plumbagin & $5 \mathrm{gm}$ & Few days & $\begin{array}{l}\text { Steroidal glycosides } \\
\text { Cardiac glycosides }\end{array}$ & {$[43]$} \\
\hline $\begin{array}{c}\text { Aconitum napellus } \\
\text { (Monkshood and } \\
\text { mitha zahar }\end{array}$ & Ran & All parts & $\begin{array}{c}\text { Aconitine, Pseudo } \\
\text { Aconite, } \\
\text { Aconine }\end{array}$ & $\begin{array}{l}1-2 \text { gram (root) } \\
1-2 \mathrm{mg} \text { Aconotine }\end{array}$ & 2 to $6 \mathrm{~h}$ & Alkaloids & {$[44,45]$} \\
\hline $\begin{array}{c}\text { Antiaris toxicaria } \\
\text { (Upas tree, Antiaris) }\end{array}$ & Mor & $\begin{array}{l}\text { Leaves and } \\
\text { bark }\end{array}$ & $\alpha$ - Antiarin & $\begin{array}{c}\mathrm{LD}-0.116 \mathrm{mg} / \mathrm{kg} \text { i.v. } \alpha- \\
\text { antiarin }\end{array}$ & $2-3$ days & Glycoside & [46] \\
\hline $\begin{array}{c}\text { Myristica fragrans } \\
\text { (Nutmeg, Mace tree) }\end{array}$ & Myr & Seeds & Myristicin and Elemicin & \begin{tabular}{|c|}
$1-3$ nut-megs(adult) \\
2 nutmegs (child) \\
\end{tabular} & $3-6 h$ & Alkaloids & {$[47,48]$} \\
\hline $\begin{array}{l}\text { Prunus amygdalus } \\
\text { (Almond, Badam) }\end{array}$ & Ros & Almond & Amygdalin & $\begin{array}{l}20 \text { almonds (adult) } \\
10 \text { almonds (Child) }\end{array}$ & & $\begin{array}{l}\text { Cyanophoric glycosides } \\
\text { Cyanogenetic glycosides }\end{array}$ & [49] \\
\hline $\begin{array}{c}\text { Alocasia } \\
\text { macrorrhiza } \\
\text { (Giant taro, Elephant } \\
\text { ear) } \\
\end{array}$ & Ara & All parts & $\begin{array}{l}\text { Cyanogenic Glycosides } \\
\text { and Calcium oxalate }\end{array}$ & $\begin{array}{c}1 / 30 \text { to } 1 / 15 \text { of } \\
\text { a grain }\end{array}$ & $15 \mathrm{~min}$ & Cyanophoric glycosides & {$[50,51]$} \\
\hline $\begin{array}{c}\text { Gossypium sp. } \\
\text { (Kapas) }\end{array}$ & Mal & Seed oil & Gossypol & $2.57 \mathrm{~g} / \mathrm{kg}$ (Rat) & & Phenolic glycosides & {$[52]$} \\
\hline $\begin{array}{c}\text { Ochrocarpus } \\
\text { longifolius } \\
\text { (Naag kesar) }\end{array}$ & Gut & All parts & $\begin{array}{c}\text { Surangin A } \\
\text { and Surangin B }\end{array}$ & $\begin{array}{c}\mathrm{LD}-9 \mathrm{mg} / \mathrm{kg} \\
\text { surangin } \mathrm{A} \\
1 \mathrm{mg} / \mathrm{kg} \text { surangin } \mathrm{B} \\
\text { (Cats }\end{array}$ & & Alkaloids & [53] \\
\hline $\begin{array}{l}\text { Peganum harmala } \\
\text { (Wild rue) }\end{array}$ & Zyg & All parts & $\begin{array}{c}\text { Harmaline, Harmine, } \\
\text { Harmane, } \\
\text { Harmalol, } \\
\text { Vasicinone } \\
\end{array}$ & $\begin{array}{l}\text { Harmaline-120 mg/kg } \\
\text { (rat), } \\
\text { Harman-200 mg/kg } \\
\text { (rabbit) }\end{array}$ & & Alkaloids & {$[54,55]$} \\
\hline $\begin{array}{l}\text { Strychnos nux- } \\
\text { vomica } \\
\text { (Poison nut and } \\
\text { kuchila) }\end{array}$ & $\log$ & $\begin{array}{l}\text { All parts } \\
\text { especially } \\
\text { seeds }\end{array}$ & $\begin{array}{c}\text { Strychnine, Brucine and } \\
\text { Vomicine }\end{array}$ & $\begin{array}{l}15-20 \mathrm{mg} / \mathrm{kg} \\
(1 \text { seed }- \text { Oral })\end{array}$ & $1-2 h$ & Indole alkaloids. & {$[56,57]$} \\
\hline $\begin{array}{l}\text { Cinchona officinalis } \\
\text { (Cinchona) }\end{array}$ & Rub & Bark & $\begin{array}{l}\text { Quinine, Cinchonine } \\
\text { and Cinch-onidine }\end{array}$ & $8-10 \mathrm{~g}$ & $\begin{array}{l}2 \mathrm{~h} \text { to } 2 \\
\text { days }\end{array}$ & Quinoline alkaloids & {$[58]$} \\
\hline $\begin{array}{c}\text { Gloriosa superba } \\
\text { (Flame lily and } \\
\text { Kalihari) }\end{array}$ & Lili & $\begin{array}{l}\text { Tubers and } \\
\text { roots }\end{array}$ & $\begin{array}{l}\text { Colchicine, } \\
\text { Gloriosine }\end{array}$ & $6 \mathrm{mg} / \mathrm{kg}$ & $12-72 \mathrm{~h}$ & Alkaloidal amines & [59] \\
\hline
\end{tabular}




\begin{tabular}{|c|c|c|c|c|c|c|c|}
\hline $\begin{array}{l}\text { Semecarpus } \\
\text { anacardium } \\
\text { (Marking nut } \\
\text { And bhilawa) }\end{array}$ & Ana & Juice & $\begin{array}{l}\text { Semecarpol and } \\
\text { Bhilawanol }\end{array}$ & $5-10 \mathrm{gm}$ & $12-24 \mathrm{~h}$ & Flavonol glycosides & [62] \\
\hline $\begin{array}{l}\text { Cannabis sativa } \\
\text { (Indian hemp } \\
\text { Hashish) }\end{array}$ & Cann & \begin{tabular}{|c|} 
Bhang, \\
Ganja- flowers \\
Charas- resin \\
leaves stems \\
\end{tabular} & Cannabin, Cannabinol & $\begin{array}{c}10 \text { gm/kg b.wt.-bhang, } \\
8 \text { gm-ganja, } \\
2 \text { gram-charas }\end{array}$ & 5-8 Days & Cannabidiolic acid & {$[63,64]$} \\
\hline $\begin{array}{l}\text { Erythroxylum coca } \\
\quad \text { (Coke, snow) }\end{array}$ & Lin & Leaves & $\begin{array}{c}\text { Cocaine, Procaine, } \\
\text { Butacaine and } \\
\text { Dibucaine }\end{array}$ & 1 - 1.5 g Cocaine (Oral) & $\begin{array}{l}15 \mathrm{~min} \text { to } \\
10 \mathrm{~h}\end{array}$ & Tropane alkaloid & [65] \\
\hline $\begin{array}{l}\text { Citrullus Colocynthis } \\
\text { (bitter cucumber) }\end{array}$ & $\mathrm{Cuc}$ & $\begin{array}{l}\text { Fruit, root, } \\
\text { dripped pulp }\end{array}$ & $\begin{array}{l}\text { Cucurbitacins } \\
\text { Saponins } \\
\text { colocynthin }\end{array}$ & $\begin{array}{l}1.3 \mathrm{~g} / \mathrm{kg} \\
\text { (rats) }\end{array}$ & & $\begin{array}{c}\text { Triterpene alkaloids } \\
\text { Glycosides }\end{array}$ & {$[66,67]$} \\
\hline $\begin{array}{l}\text { Conium macukatum } \\
\text { (Poison hemlock) }\end{array}$ & Api & All parts & $\begin{array}{c}\text { Coniine } \\
\text { N-methylconiine } \\
\text { conhydrine }\end{array}$ & $\begin{array}{l}0.1 \mathrm{~g} \text { of coniine }(1 \mathrm{seed} \\
\text { oral) }\end{array}$ & & Piperidine alkaloids & [68] \\
\hline
\end{tabular}

*(Families: Eup (Euphorbiaceae), Sola (Solanaceae), Apocy (Apocyanacae), Legu (leguminosae), Faba (Fabaceae), Papa (Papaveraceae), Cam (Campanulaceae), Plu (Plumbaginaceae), Ran (Ranunculaceae), Mor (Moraceae), Myr (Myristicaceae), Ros (Rosaceae), Ara (Araceae), Mal (Malvaceae), Gut (Gutiferae), Zyg (Zygophyllaceae), Log (Loganiaceae), Rub (Rubiaceae), Lili (Liliaceae), Ter (Terophularaceae), Ana (Anacardiaceae), Cann (Cannabinaceae), Lin (Linaceae), Cuc (Cucurbitaceae), Api (Apiaceae)

\section{Chemical classification of toxic phytochemicals}

The chemical constituent of plants formed through their cell activity is organic in nature. Each plant has enzymes that convert simple substances into complex compounds. It is necessary to be aware of the fact that overdose of plant extracts can cause harmful effects ${ }^{[68]}$. The chemical constituent of toxic plants are alkaloids, glycosides, proteins, resins etc. Depending upon their chemical composition, toxic plants are mainly categorized into four groups, which are further subdivided on the basis of their pharmacological action and chemical structure.

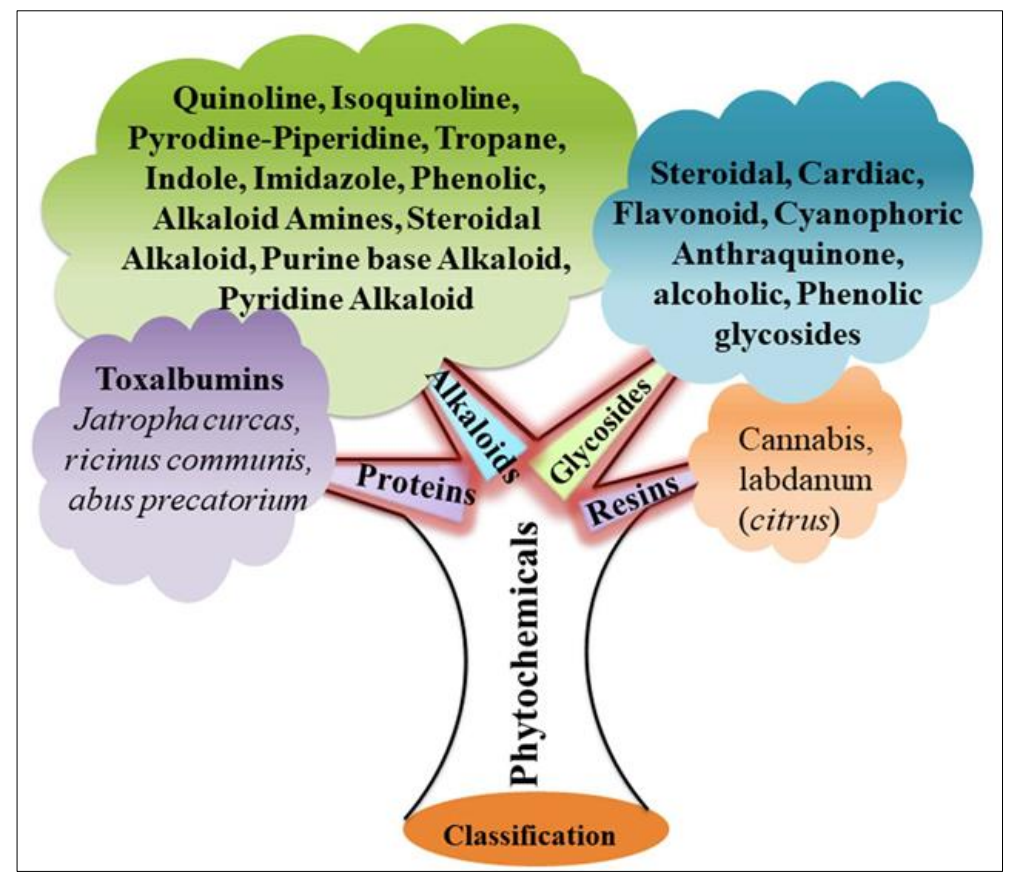

Fig 2: The classification of phytochemical present in Indian plants on the basis of there chemical components and active constituents

\subsection{Alkaloids}

Alkaloids are heterocyclic organic compounds containing nitrogen atoms and mainly produced by plants. Some of the alkaloids are not present in the heterocyclic form such as ephedrine, mescaline, colchicine, hordenine. The alkaloids are found in various forms like root (Rauwolfia), seed (Strychrios), leaves (Belladonna), fruits (Piper). The alkaloid includes $\alpha$ propyl piperidine, pyridine- piperidine and derivative of nicotinic acid (Nicotine, Labaline, Piperidine, Ricinine). Quinoline is a basic ring structure obtained from the barks of cinchona species (Cinchona officinalis) contains cinchonine, cuspareine. Isoquinoline structure of alkaloids obtained from
Papaver somniferum, Argemone mexicana (opium, morphine, codeine, thebaine) contains benzylisoquinoline, papaverine, narcotine, narceine. Tropane is a bicyclic structure that contains tropic acid, benzoic acid (Belladonna, Hyoscyamme, cocaine). Indole nucleus of alkaloid chemical structure is obtained from Rauwolfia, Vinca, Ergot, Nux vomica, Brucine. Steroidal alkaloids containing the imidazole ring represents by pilocarpus (phenanthrene group). Alkaloid amines contain derivatives of phenylethylamine (Ephedra, Colchium corn). Senecio alkaloids have heterocyclic rings of pyrrolizidine (Seneciohylline). Purine bases as nucleus structure is present in caffeine, theophylline and theobromine (tea leaves, coffee seeds). 


\subsection{Glycosides}

The condensation product of sugar (including polysaccharides) and non-sugar parts of glycosides (aglycone) in form of Steroidal glycosides, Cardiac glycosides, Flavonoid glycosides, Anthraquinone glycosides, Cyanophoric glycosides. The glycosides consist of hydrogen, carbon, oxygen (some contain nitrogen and sulfur) ${ }^{[69]}$. The yellowcolored pigments called flavinoid glycosides are widely distributed in higher plants contain rutin, quercetin and citrus (bioflavonoids). The steroidal, cardiac glycosides are present in Calotropis, Oleander, Strophanthus and Convallana. The orange-colored pigments called anthraquinone glycosides, contain derivatives of polyhydroxy -anthraquinone. On hydrolysis, hydrocyanic acid produces cyanogenic glycoside (Brassica alba). Glucovanillin and glucovanillin alcohol on hydrolysis forms glucose and vanillic acid, after oxidation it converts into vanillic aldehyde. Phenolic glycosides (Arbutin) are found in Chimaphila, a citrus fruit, and in the barks of Rosaceous plants.

\subsection{Toxic proteins}

Jatropha curcas, Ricinus communis, Abrus precatorius, and Croton tiglum are some of the common plants containing a toxic protein called toxalbumins. These Toxalbumins leads to precipitation and agglutination of red blood cells. The toxic protein of plants comes from the group of phytotoxins, which modifies the $28 \mathrm{~S}$ rRNA-60S ribosomal subunits and protein synthesis is also affected in eukaryotic cells.

\subsection{Resin}

Resin is a semisolid organic substance secreted by most of the plants composed mainly of terpenes and its derivatives. Some resins also contain a large number of resin acids e.g., hashish (Cannabis resin) from Cannabis indica, labdanum from the Mediterranean species of Cistus. The seeds of Ricinus communis plants are highly toxic in nature. Triglyceride of ricinoleic acid and purgative oil are incorporated in the seeds of Ricinus plants.

Table 2: Show the chemical structure of active chemicals constituent of different class of alkaloids and glycosides founds in plants Alkaloids

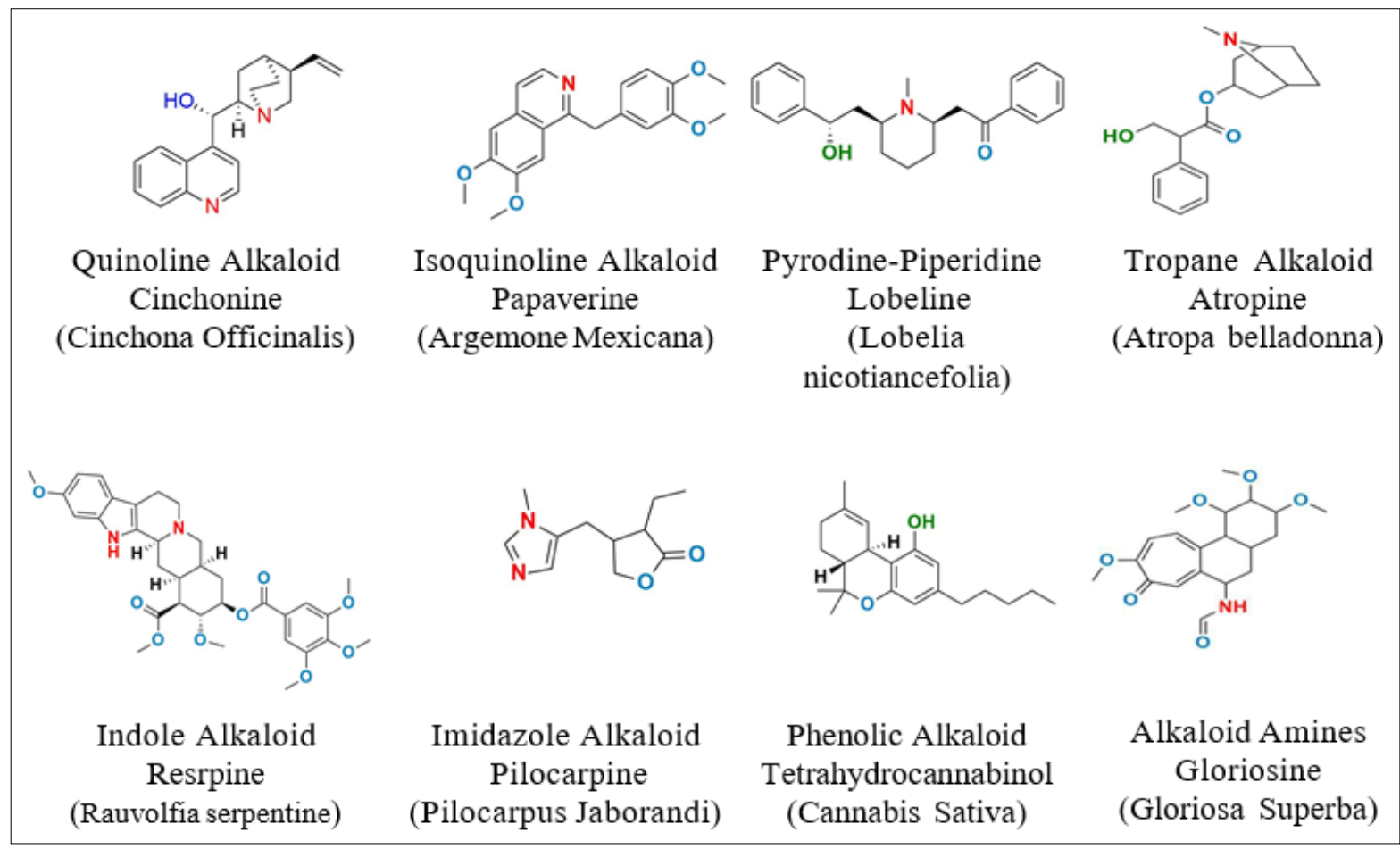<smiles>COc1ccc(C(=O)Cc2ccc(OC)c(OC)c2C(=O)O)c(OC)c1CCN(C)C</smiles>

Steroidal Alkaloid

Narceine (Papaver Somniferum)<smiles>Cn1c(=O)[nH]c2ncn(C)c2c1=O</smiles>

Purine base Alkaloid

Caffeine

(Rubiaceae)<smiles>C=CCc1cc(OC)c2c(c1)OCO2</smiles>

Steroidal Alkaloid

Myristicin (Myristica fragrans)<smiles>CN1CCCC1c1cccnc1</smiles>

Pyridine Alkaloid Nicotine

(Nicotiana tabacum)

Glycosides 
<smiles>N#CCC(N)C(=O)O</smiles>

\section{Cyanophoric Glycoside Cyanoanaline (Lathyrus sativus)}<smiles>CC(=O)OC1C[C@]2(O)C3CCC4CCCCC4(C)C3CC[C@]2(C)[C@H]1C1COC(=O)C1</smiles>

Cardaric Glycoside Neriodin (Nerium odorum)

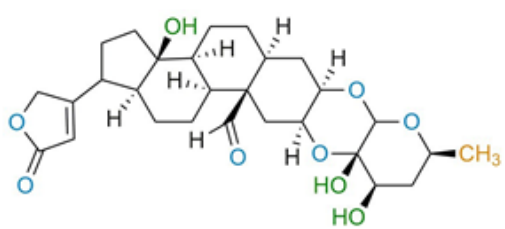

Cardiac Glycoside
Calotropin
(Calotropis gigantea)<smiles>CC(C)(C#N)OC1OC(CO)C(O)C(O)C1O</smiles>

Cyanogenetic Glycoside Myristicin (Manihot utilissima)

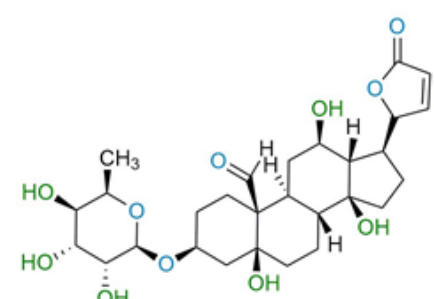

Steroidal glycosides $\alpha$-Antiarin (Antiaris toxicaria)<smiles>CO[C@H]1C(OC(C)=O)C(OC2CC[C@H]3C4CCC5C(C6=CC(=O)OC6)CCC5(O)C4CC[C@]3(C)C2)OC(C)[C@H]1O</smiles>

Steroidal glycosides Cerberin (Cerbara odollum)

\section{Metabolic effect of Toxic chemical constituent on the living organism \\ 3.1 Cardiovascular system}

Two healthy patients ingested extract of Celeistanthus collinus to commit suicide. It was found that both patients were affected by severe complications such as cardiac arrhythmias, hypokalemia, hypotension, and neuromuscular weakness ${ }^{[70]}$. The fruit of Citrus Aurantivam contains Synephrine which has adverse cardiovascular effects (hypertension, cardiac arrhythmias) ${ }^{[71]}$. Cannabis (Cannabis sativa) causes a multiorgan effect on peripheral vascular, cerebrovascular system and occurrence of arrhythmias, increase the risk of myocardial infarction and atrial fibrillation ${ }^{[72]}$. A 60-year-old man consumed the seeds of Thevetia nereifolia that caused cardiovascular symptoms. About an hour after ingestion, there were major changes his body including ECG change, sweating, burning sensation, diarrhea, and vomiting ${ }^{[73]}$.

\subsection{Effect on Central Nervous System}

The leaf extract of Cerbera Odollam (Apocynaceae) contains cerberin which causes disruption in heartbeat. It is also found in the study that it decreases motor activity in mice. It reduces the effective duration of pentylenetetrazole induced tonic seizures, mortality and generates a hypnotic affect ${ }^{[74]}$. Root parts of Calotropis gigantea extract effect on locomotors activity and has sedative, analgesic effects on albino rats at the dose of $250-500 \mathrm{mg} / \mathrm{kg}$ bodyweight ${ }^{[75]}$. All parts of Atropa belladonna contain atropine alkaloids which cause anticholinergic syndrome, disorientation, short-term memory loss, confusion and psychosis [76]. The overdose of Datura fastuosa leaf extract serves hallucinogenic effect and cause

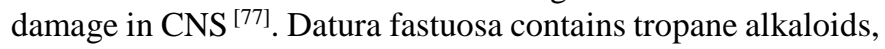
even small dose can cause acute confusion, fever, tachycardia, dilated pupils, urinary retention, delirium, rapid and weak pulse.

\subsection{Gastrointestinal effect}

The toxic protein of Croton seeds causes gastrointestinal edema by increasing gastrointestinal permeability ${ }^{[78]}$. Fruit of
Jatropha multifida containing toxalbumin resin, cause dehydration, vomiting, renal and hepatic impairment [79]. Pathology revealed that castor beans (Ricinus communis) inhibit protein synthesis, cause gastroenteritis, hepatic necrosis and acute tubular necrosis in kidney ${ }^{[80]}$. It is now known that alkaloids of the Rauwolfia serpentine effect proximal tube cells of the kidney ${ }^{[81]}$. Extracts of croton oil, castor oil, colocynth, juice of calotropis cause irritation of colon may produce hyperemia and contraction of uterus can cause abortion.

\subsection{Effect on other body parts}

Abrin of Abrus precatorius contains two subunits of protein $A$ and B. $0.1-1 \mu \mathrm{g} / \mathrm{kg}$ fatal dose of protein A and B inhibit protein synthesis by inactivating $26 \mathrm{~S}$ subunit of the ribosome. The ingestion of leaves, seeds and sprouts of Aesculus hippocastacanum effects on muscle system and may cause paralysis, dilated pupils and stupor ${ }^{[83]}$. The Cleistanthus collinus leaves cause cardiac arrhythmias ${ }^{[84]}$ hypertension, hypoxia ${ }^{[85]}$, vasodilation ${ }^{[86-88]}$ neuromuscular weakness. The fatal period of 6 hours with a dose of 20-40ml of Aconitum ferox extract has a severe effect on gastrointestinal and cardiovascular organs. It causes a sign of vomiting, diarrhea, burning sensation, sweating, headache and confusion. It may cause the death of a person due to ventricular arrhythmias, paralysis of the heart ${ }^{[89]}$. Gloriosa superba has a high content of colchicine and it is used to commit suicide, murder and to kill animals ${ }^{[90]}$. After a few hours of ingestion, the person may experience vomiting, electrolyte imbalance, respiratory distress abdomen pain and burning in the throat ${ }^{[91]}$.

\section{Conclusion}

In the present review, the author reported more than 70 poisonous plant species belonging to different families. The majority of the active constituent of poisonous parts comes from roots, latex, barks, and seeds. Besides these, the minority of poisons comes from parts of fruits, tubers, bulbs and sometimes whole plants. This report also contains toxic constituent and their metabolic effects on human beings as well as livestock. Significance of this review article on such toxic 
plants provides helpful information for forensic investigators. Further, these active chemical constituents are found in viscera as well as in the crime scene. On the basis of plants active constituent found in viscera, forensic experts can predicate whether it is a case of suicide, homicide or accidental with the help of this article.

\section{Acknowledgement}

I humbly acknowledge Dr. S.K. Pandey, Associate Professor, Department of Forensic Science, Banaras Hindu University for sharing their pearls of wisdom during course of writing article. I am also gracefully acknowledge Mr. R. Verma (CSIR-IITR), Dr. Kamalakshi Krishanamurthy (Director I/C retire) at Forensic Science department, Chennai, Tamil Nadu, S. Prakash, S. Agrawal, SSA, Toxicology Division, Regional Forensic Science Laboratory (Chanykyapuri, New Delhi, India) for every support and guidance.

\section{References}

1. Laboratory Procedure Manual- Forensic Toxicology, Directorate of Forensic Science, MHA, Govt. of India.

2. Biswas G. Review of Forensic Medicine and Toxicology, the Health Sciences Publisher, New Delhi 2010.

3. Ballantyne B, Mars TC, Turner P. Fundamentals of toxicology. General and Applied Toxicology. New York: McMillan Press 1995.

4. Narayan Reddy KS. Medical Jurisprudence and Toxicology (Law Practice \& Procedure). Hyderabad: ALT Publications 2005.

5. Khajja BS, Sharma M, Singh R, Mathur GK. Forensic study of Indian toxicological plants as botanical weapon (BW): A review. J Environ Anal Toxicol 2011;(1):112.

6. Qureshi JM, Bano S, Mohammad T, Khan MA. Medicinal potential of poisonous plants of tehsil Kahuta from district Rawalpindi, Pakistan. Pak J Biol Sci 2001;(4):331-332.

7. Greval SD. Lyon's Medical Jurisprudence for India. 10th ed. Law Publishers (India) Pvt. Ltd: DLH 1953.

8. Viswanathan N, Joshi BS. Toxic Constituents on Some Indian Plants. Curr Sci 1983;52:1.

9. Kesri R, Joseph J, Henry RA, Thomas A, Rao GG. Cleistanthus collinus poisoning; Diagnostic dilemma and n-acetyl cysteine as a possible treatment option. J Assoc Physicians India 2016;64(1):142.

10. Mohan A, Naik GS, Harikrishna J, Kumar DP, Rao MH et al. Cleistanthus collinus poisoning: experience at a medical intensive care unit in a tertiary care hospital in south India. Indian J Med Res 2016;143(6):793-797.

11. Fernando K, Jayasekara K, Warushahennadi J, Kumarasinghe I, Weerakoon $\mathrm{K}$ et al. Intentional ingestion of Strychnos nux-vomica seeds causing severe muscle spasms and cardiac arrest: a postmortem report. Wilderness Environ Med 2015;26(1):101-102.

12. Schmidt RJ, Evans FJ. Skin irritants of the sun spurge (Euphorbia helioscopia L.). Contact Dermatitis 1980;6(3):204-210.

13. Zayed SM, Farghaly M, Soliman SM, Gotta H, Sorg B et al. Dietary cancer risk from conditional cancerogens (tumor promoters) in produce of livestock fed on species of spurge (Euphorbiaceae). V. Skin irriitant and tumorpromoting diterpene ester toxins of the tigliane and ingenane type in the herbs Euphorbia nubica and Euphorbia helioscopia contaminating fodder of livestock. J Cancer Res Clin Oncol 2001;127(1):40-47.
14. Taylor P, Devappa RK, Makkar HPS, Becker K. Journal of Toxicology and Environmental Health, Part B: Critical Reviews Jatropha Toxicity: A Review. (November 2013) 2010, https://doi.org/10.1080/10937404.2010.499736

15. Soto-blanco B, GSL. Toxic effects of prolonged administration of leaves of cassava (Manihot esculenta Crantz) to goats 2010;62:361-366. https://doi.org/10.1016/j.etp.2009.05.011

16. Onwuka CFI. Hydrocyanic acid contents of tropical browse and their influence on performance of goats 1992;45:5-10.

17. Martínez MA, Martínez SL, Margarit SA, Trenchs SMV, Luaces CC. Poisonous plants: An ongoing problem. An Pediatr (Barc) 2015;82(5):347-353.

18. Wang CF, Nie XJ, Chen GM, Yu ZH, Li Z et al. Early plasma exchange for treating ricin toxicity in children after castor bean ingestion. J Clin Apher 2015;30(3):141-146.

19. Demirhan A, Tekelioğlu ÜY, Yıldız İ, Korkmaz T, Bilgi $\mathrm{M}$ et al. Anticholinergic Toxic Syndrome Caused by Atropa Belladonna Fruit (Deadly Nightshade): A Case Report. Turk J Anaesthesiol Reanim 2013;41(6):226-228.

20. Glatstein M, Danino D, Wolyniez I, Scolnik D. Seizures caused by ingestion of Atropa belladonna in a homeopathic medicine in a previously well infant: case report and review of the literature. Am $\mathrm{J}$ Ther 2014;21(6):e196-e198.

21. Soto-blanco B, GSL. Toxic effects of prolonged administration of leaves of cassava (Manihot esculenta Crantz) to goats 2010;62:361-366. https://doi.org/10.1016/j.etp.2009.05.011

22. Quek KC, Cheah JS. Poisoning due to ingestion of the seeds of kechubong (Datura fastuosa) for its ganja-like effect in Singapore. J Trop Med Hyg 1974;77(5):111-112.

23. Hodjat M, Rezvanfar MA, Abdollahi M. A systematic review on the role of environmental toxicants in stem cells aging. Food Chem Toxicol 2015;86:298-308.

24. Demirhan A, Tekelioğlu ÜY, Yıldız İ, Korkmaz T, Bilgi $\mathrm{M}$ et al. Anticholinergic Toxic Syndrome Caused by Atropa Belladonna Fruit (Deadly Nightshade): A Case Report. Turk J Anaesthesiol Reanim 2013;41(6):226-228.

25. Slotkin TA, Skavicus S, Card J, Stadler A, Levin ED et al. Developmental Neurotoxicity of Tobacco Smoke Directed Toward Cholinergic and Serotonergic Systems: More than Just Nicotine. Toxicol Sci 2015;147(1):178-189.

26. Notes C, Of C, By P, Nigrum S. Clinical notes. A case of poisoning by solanum nigrum 1952, 77-80.

27. Mahesh Hadimani, Seema, MG A. A review on toxicity of calotropis (ARKA) and Management. International Journal of Ayurveda and Pharma Research 2015, 3(4). Retrieved from https://ijapr.in/index.php/ijapr/article/view/139

28. Narendranathan M, Krishna DKV, Vijayaraghavan G. Eelectrocardiographic changes in cerbera odollum poisoning. J Assoc Physicians India 1975;23(11):757-762.

29. de Lima JM, de Freitas FJ, Amorim RN, Câmara AC, Batista JS et al. Clinical and pathological effects of Calotropis procera exposure in sheep and rats. Toxicon 2011;57(1):183-185.

30. Bandara V, Weinstein SA, White J, Eddleston M. A review of the natural history, toxinology, diagnosis and clinical management of Nerium oleander (common oleander) and Thevetia peruviana (yellow oleander) poisoning. Toxicon 2010;56(3):273-281. 
31. Gicquel T, Lepage S, Baert A, Bouvet R, Morel I. Death related to consumption of Rauvolfia sp. Powder mislabeled as Tabernanthe iboga 2016, 6-10. https://doi.org/10.1016/j.forsciint.2016.06.014

32. Boor PJ. Oleander toxicity : an examination of human and animal toxic exposures 1996, 109.

33. Alfonso HA, Sanchez LM, Figeurdo MA, Gomez BC. Intoxication due to Crotalaria retusa and $\mathrm{C}$ spectabilis in chickens and geese. Vet Hum Toxicol 1993;35(6):539.

34. Abascal K. Botanicals for Regulating Heart Rhythms 2000.

35. Suresh S, Prithiviraj E, Prakash S. Dose- and timedependent effects of ethanolic extract of Mucuna pruriens Linn. Seed on sexual behaviour of normal male rats 2009; 122:497-501. https://doi.org/10.1016/j.jep.2009.01.032

36. Patil MM, Patil SV, Akki AS, Lakhkar B, Badiger S. An Arrow Poison (Abrus Precatorius) Causing Fatal Poisoning in a Child. J Clin Diagn Res 2016;10(3):SD03SD04.

37. Powers JJ, May KN, Rheins MS, Harvey SC, Smith RC, Dwyer CM et al. Somatic Variation of Red Cell Agglutinogens Due to Hormonal Influence 1960, 14.

38. Babu CK, Ansari KM, Mehrotra S, Patel S, Dikshit M et al. Activation of inflammatory response and apoptosis of polymorphonuclear leukocytes in patients with argemone oil poisoning. Chem Biol Interact 2010;183(1):154-164.

39. Babu CK, Khanna SK, Das M. Antioxidant status of erythrocytes and their response to oxidative challenge in humans with argemone oil poisoning. Toxicol Appl Pharmacol 2008;230(3):304-311.

40. Babu CK, Ansari KM, Mehrotra S, Khanna R, Khanna SK et al. Alterations in redox potential of glutathione/glutathione disulfide and cysteine/cysteine disulfide couples in plasma of dropsy patients with argemone oil poisoning. Food Chem Toxicol 2008;46(7):2409-2414.

41. Carod Artal FJ. Neurological syndromes associated with the ingestion of plants and fungi with a toxic component (II). Hallucinogenic fungi and plants, mycotoxins and medicinal herbs. Rev Neurol 2003;36(10):951-960.

42. Chothani DL, Vaghasiya HU. A review on Balanites aegyptiaca Del (desert date): phytochemical constituents, traditional uses, and pharmacological activity 2011, 5(9). https://doi.org/10.4103/0973-7847.79100

43. Solomon FE, Sharada AC, Devi PU. Toxic effects of crude root extract of Plumbago rosea (Rakta chitraka) on mice and rats. J Ethnopharmacol 1993;38(1):79-84.

44. Ge YB, Jiang Y, Zhou H, Zheng M, Li J et al. Antitoxic effect of Veratrilla baillonii on the acute toxicity in mice induced by Aconitum brachypodum, one of the genus Aconitum. J Ethnopharmacol 2016;179:27-37.

45. Chan TY. Aconitum Alkaloid Poisoning Because of Contamination of Herbs by Aconite Roots. Phytother Res 2016;30(1):3-8.

46. Chuan TK, Han CW. A toxicological study of Antiaris toxicaria (Nuchientzu). Chin Med J 1949;67(5):261-264.

47. Flam B, Bendz E, Jonsson Fagerlund M, Höjer J. Seizures associated with intentional severe nutmeg intoxication. Clin Toxicol (Phila) 2015;53(9):917.

48. Sein Anand J, Barwina M, Waldman W. Acute intoxication with nutmeg used as a recreational purpose-a case report. Przegl Lek 2013;70(8):693-694.

49. Pack WK, Raudonat HW, Schmidt K. Lethal poisoning with hydrocyanic acid after ingestion of bitter almonds (Prunus amygdalus). Z Rechtsmed 1972;70(1):53-54.

50. Chan TY, Chan LY, Tam LS, Critchley JA. Neurotoxicity following the ingestion of a Chinese medicinal plant, Alocasia macrorrhiza. Hum Exp Toxicol 1995;14(9):727728.

51. Lin TJ, Hung DZ, Hu WH, Yang DY, Wu TC et al. Calcium oxalate is the main toxic component in clinical presentations of Alocasis macrorrhiza (L) Schott and Endl poisonings. Vet Hum Toxicol 1998;40(2):93-95.

52. Biology C. Effects of an aqueous extract of cotton seed (Gossypium barbadense Linn) on adult male rats 1991;7:353-362.

53. Kanyanga C, Wembonyama L, Muganza M, Maya M, Kabangu K. Effects of the lyophilized aqueous extract form the root bark of perianthus longifolia miers (menispermaceae ) on sexual behaviors of normal 2016;5(4):135-155.

Https://doi.org/10.20959/wjpps20164-6278

54. Narasimhan TR, Ananth M, Swamy MN, Babu MR, Mangala A et al. Toxicity of Parthenium hysterophorus L. to cattle and buffaloes. Experientia 1977;33(10):13581359.

55. Sadr Mohammadi R, Bidaki R, Mirdrikvand F, Mostafavi Yazdi SN, Yazdian Anari P. Peganum Harmala (Aspand) Intoxication; a Case Report. Emerg (Tehran) 2016;4(2):106-107.

56. Prat S, Hoizey G, Lefrancq T, Saint-Martin P. An unusual case of strychnine poisoning. J Forensic Sci 2015;60(3):816-817.

57. Fernando K, Jayasekara K, Warushahennadi J, Kumarasinghe I, Weerakoon $\mathrm{K}$ et al. Intentional ingestion of Strychnos nux-vomica seeds causing severe muscle spasms and cardiac arrest: a postmortem report. Wilderness Environ Med 2015;26(1):101-102.

58. Gurung P, De P. Spectrum of biological properties of cinchona alkaloids: A brief review 2017;6(4):162-166.

59. Kande VCJ, Ekanayeka R, Wijewardane DK. Case report: a rare case of attempted homicide with Gloriosa superba seeds. BMC Pharmacol Toxicol 2016;17(1):26.

60. Lin CC, Yang CC, Phua DH, Deng JF, Lu LH. An outbreak of fox glove leaf poisoning. J Chin Med Assoc 2010;73(2):97-100.

61. Vyas A, Bachani N, Thakur H, Lokhandwala Y. Digitalis toxicity: ECG vignette. Indian Heart J 2016;68(2):S223S225.

62. Matthai TP, Date A. Renal cortical necrosis following exposure to sap of the marking- nut tree (Semecarpus anacardium). Am J Trop Med Hyg 1979;28(4):773-774.

63. Hamilton I. The need for health warnings about cannabis and psychosis. Lancet Psychiatry 2016;3(3):188-189.

64. Rabner J, Gottlieb S, Lazdowsky L, LeBel A. Psychosis following traumatic brain injury and cannabis use in late adolescence. Am J Addict 2016;25(2):91-93.

65. Zimmerman JL. Cocaine intoxication. Crit Care Clin 2012;28(4):517-526.

66. Al-Yahya MA, AL-Farhan AH, Adam SE. Preliminary toxicity study on the individual and combined effects of Citrullus colocynthis and Nerium oleander in rats. Fitoterapia 2000;71(4):385-391.

67. Pfab R. Poisoning by Citrullus colocynthis. Unknown to us, a frequent poisonous plant in foreign travel. MMW Fortschr Med 1999;141(31-32):41-42.

68. Chopra RN, Bhadwar RL, Ghosh S. Poisonous Plants 
of India. Jaipur, India: Academic Publishers, 1984.

69. Siwach SB, Gupta A. The profile of acute poisonings in Harayana- Rohtak Study. J Assoc Physicians India 1995;43:756-9.

70. Eswarappa S, Chakraborty AR, Palatty BU, Vasnaik M. Cleistanthus collinus Poisoning: Case Reports and Review of the Literature 2003;41(4):369-372. https://doi.org/10.1081/CLT-120022005

71. Calapai G, Firenzuoli F, Squadrito F, Arlotta MR. Antiobesity and cardiovascular toxic effects of Citrus aurantium extracts in the rat: A preliminary report 1999, 586-592.

72. Pratap B, Korniyenko A. Toxic Effects of Marijuana on the Cardiovascular System. (December 2011) 2012, 143-148. https://doi.org/10.1007/s12012-011-9150-y

73. Maringhini G, Notaro L, Barberi O, Giubilato A, Butera $\mathrm{R}$ (n.d.). Cardiovascular glycoside-like intoxication following ingestion of Thevetia nereifolia/peruviana seeds: A case report 2012, 2-5.

74. Navarro-delmasure C. Toxicity and effects on the central nervous system of a Cerbera odollam leaf extract 1991;34:201-206.

75. Argal A, Pathak AK. CNS activity of Calotropis gigantea roots https://doi.org/10.1016/j.jep.2005.12.024

76. Access O. Case report 2012;8688:2-5.

77. Ratan L, Article R, Anju D, Ratan L. Available online through 2011;2(1):145-150.

78. Science A, Vol L. Gastrointestinal effects of croton tiglium in experimental animals N.R. Pillai 1999;18(18):14.

79. Multifida J. Selected Topics 2000;19(2):173-175.

80. Aslani MR, Maleki M, Mohri M, Sharifi K, Najjar-nezhad $\mathrm{V}$, Afshari E. Castor bean (Ricinus communis) toxicosis in a sheep flock 2007;49:400-406. https://doi.org/10.1016/j.toxicon.2006.10.010

81. Mossoba ME, Flynn TJ, Vohra S, Wiesenfeld PL, Sprando RL. Human kidney proximal tubule cells are vulnerable to the effects of Rauwolfia serpentina 2015-2016, 285-293. https://doi.org/10.1007/s10565-016-9311-7

82. Tamilselvan N, Thirumalai T, Shyamala P, David E. A review on some poisonous plants and their medicinal values. Journal of Acute Disease 2014;3(2):85-89. https://doi.org/10.1016/S2221-6189(14)60022-6

83. Aesculus hippocastanum [Online]. Available from: http://www.ces.ncsu.edu/depts/hort/consumer/poison/ Aescuhi.htm

84. Tewari DN. Report of the Task Force on Conservation \& Sustainable use of Medicinal Plants. [Online]. Available from: http:// planningcommission.nic.in/aboutus/taskforce/tsk_medi.p df

85. Subrahmanyam DK, Mooney T, Raveendran R, Zachariah B. A clinical and laboratory profile of Cleistanthus collinus poisoning. J Assoc Physicians 2003;51:10521054.

86. Benjamin SPE, Fernando ME, Jayanth JJ, Preetha B. Cleistanthus collinus poisoning. J Assoc Physicians 2006;54:742-744.

87. Eswarappa S, Chakraborty AR, Palatty UB, Vasnik M. Cleistanthus Collinus poisoning: case reports and review of the literature. Clin Toxicol 2003;41(4):369-372.

88. Nampoothiri $K$, Chrispal A, Begum A, Jasmine $S$, Gopinath KG, Zachariah A. A clinical study of renal tubular dysfunction in Cleistanthus collinus (Oduvanthalai) poisoning. Clin Toxicol 2010;48(3):193197.

89. Chan TY. Aconite poisoning. Clin Toxicol (Phila.) 2009;47(4):279-285.

90. Fernando R, Widyaratna D. Gloriosa superba. INCHEM: International Programme on Chemical Safety (IPCS) 1989.

91. Lal HS, Mishra PK. Gloriosa superba: an endangered plant spotted for the first time from forest of Tpchanch, Hazaribag (Jharkhand) India. Sci Res Rep 2011;1(2):6164. 